\title{
Allele-specific repression of lymphotoxin- $\alpha$ by activated B cell factor-1
}

\author{
Julian C Knight ${ }^{1}$, Brendan J Keating ${ }^{1}$ \& Dominic P Kwiatkowski ${ }^{1,2}$
}

Genetic variation at the human LTA locus, encoding lymphotoxin- $\alpha$, is associated with susceptibility to myocardial infarction, asthma and other diseases. By detailed haplotypic analysis of the locus, we identified a single-nucleotide polymorphism (SNP) at $L T A+80$ as a main predictor of LTA protein production by human $B$ cells. We found that activated B-cell factor-1 (ABF-1) binds to this site in vitro and suppresses reporter gene expression, but only in the presence of the LTA+80A allele. Using haplotype-specific chromatin immunoprecipitation, we confirmed that ABF-1 is preferentially recruited to the low-producer allele in vivo. These findings provide a molecular model of how LTA expression may be genetically regulated by allele-specific recruitment of the transcriptional repressor $\mathrm{ABF}-1$.

Figure 1 LTA protein production is associated with LTA haplotype. (a) Haplotype structure at the LTA locus. SNPs present at an allele frequency $>0.1$ in the panel of $26 \mathrm{LCLs}$ are shown (minor allele frequencies given in brackets) together with haplotypes (haplotype frequencies shown at the head of the columns). Designations of SNPs refer to the nucleotide position relative to transcriptional start site. Clade $\mathrm{A}$ is uniquely defined by $\angle T A+10 \mathrm{~A}, \angle T A+252 \mathrm{G}$ and $L T A+723 A$; clade $B$ is uniquely defined by $\angle T A+80 A$ and $\angle T A+368 C$; and clade $C$ is uniquely defined by $L T A+438(10)$ and $L T A+495 C$. The frequencies of these main haplotype clades were $0.27,0.46$ and 0.27 , respectively. (b) Analysis of LTA production by haplotype pair. A scatter plot of LTA concentration in cell culture supernatant from the panel of LCLs is shown per $10^{6}$ cells, plotted as a symbol representing each cell line together with the geometric mean (horizontal lines). A total of six replicate experiments were done. The cell lines heterozygous with respect to haplotypes A, B and C are shown categorized at a given time point according to the pair of haplotypes present in that cell line $(A+B, A+C$ or $B+C)$. At $24 \mathrm{~h}$ after stimulation, cells bearing haplotypes $\mathrm{B}+\mathrm{C}$ or $\mathrm{B}+\mathrm{A}$ were associated with significantly lower LTA concentrations than those bearing haplotypes $\mathrm{A}+\mathrm{C}$ in a paired $t$-test (two-tailed; 226.7 versus $377.2, P=0.001$ and 260.5 versus $377.2, P=0.031$ for comparison of haplotypes $B+C$ with $A+C$ and of haplotypes $B+A$ with $A+C$, respectively), whereas $B+C$ versus $B+A$ showed no difference (226.7 versus 260.5, $P=0.12$ ). Similarly, after $48 \mathrm{~h}$, cells bearing haplotypes $B+C$ or $B+A$ were associated with significantly lower LTA concentrations than those bearing haplotypes $\mathrm{A}+\mathrm{C}$ in a paired $t$-test (two-tailed; 371.3 versus 557.0, $P=0.001$ and 407.7 versus 557.0, $P=0.007$ for comparison of haplotypes $B+C$ with $A+C$ and of haplotypes $B+A$ with $A+C$, respectively), whereas $B+C$ versus $\mathrm{B}+\mathrm{A}$ showed no difference (371.3 versus $407.7, P=0.25$ ).
Identifying human DNA polymorphisms that regulate gene expression is essential for understanding the genetic basis of common diseases but is technically challenging. Valuable insights into molecular mechanisms can be derived by in vitro analysis of protein-DNA binding and reporter gene expression, but we have limited tools to interrogate putative regulatory polymorphisms in vivo. Such analysis needs to consider natural chromatin structure and natural haplotypic combinations of polymorphisms. It also must be able to detect small differences in gene expression, which could be crucial for disease susceptibility if a physiologically important mediator is involved.

We attempted to address these issues for LTA, an important early mediator of immune and inflammatory responses ${ }^{1}$. Regulation of LTA is finely calibrated to resist dangerous pathogens but not to cause inflammatory damage to the host, as seems to occur in bacterial sepsis ${ }^{2}$ and

a

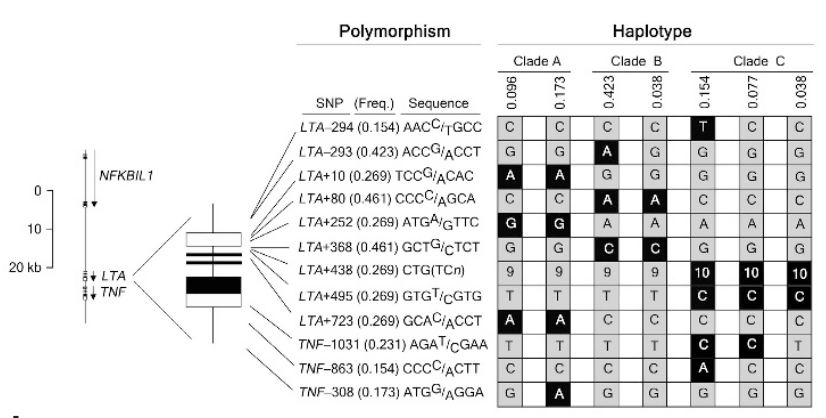

b

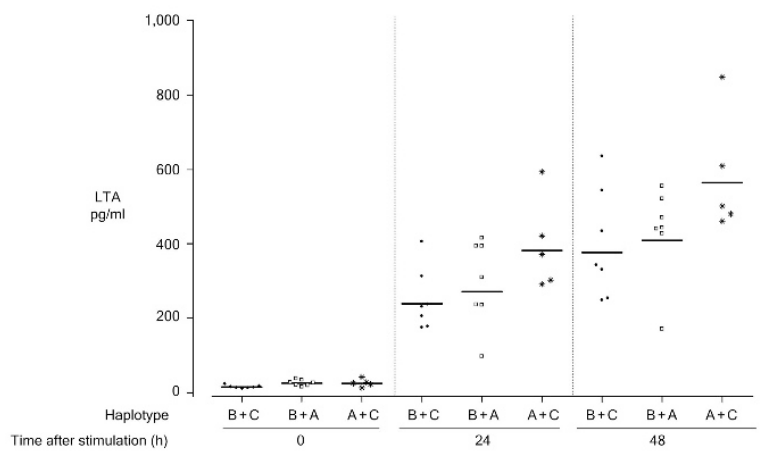

${ }^{1}$ Wellcome Trust Centre for Human Genetics, University of Oxford, Oxford OX3 7BN, UK. ${ }^{2}$ University Department of Paediatrics, John Radcliffe Hospital, Oxford OX3 9DU, UK. Correspondence should be addressed to J.C.K. (julian@well.ox.ac.uk).

Published online 28 March 2004; doi:10.1038/ng1331 
cerebral malaria ${ }^{3}$. An intronic SNP at $L T A+252$ is associated with susceptibility to myocardial infarction ${ }^{4}$, asthma ${ }^{5,6}$, bacterial sepsis ${ }^{7,8}$, cancer ${ }^{9,10}$ and autoimmune diseases ${ }^{11,12}$. Haplotypes bearing the $L T A+252 \mathrm{G}$ allele show relatively high levels of reporter gene expression ${ }^{4}$ and LTA protein production by peripheral blood mononuclear cells in some ${ }^{13,14}$ but not all $^{15,16}$ studies. An investigation of allele-specific quantification of transcripts and phosphorylated polymerase II loading was consistent with high LTA expression by haplotypes carrying the $L T A+252 \mathrm{G}$ allele ${ }^{17}$. Inspection of the data suggests that the main effect did not reside with the $L T A+252 \mathrm{G}$ allele itself, however, but rather in some other difference between the most common haplotype, which did not include the $L T A+252 \mathrm{G}$ allele, and the haplotype containing this allele.

To investigate the relationship between genotypic variation at LTA and phenotype, we first determined the sequence diversity at the locus by cloning and sequencing the gene and its flanking regions in a panel of lymphoblastoid cell lines (LCLs). We previously reported an analysis of LTA haplotypes in five individuals who were heterozygous with respect to $L T A+252$ (ref. 17). We now sequenced 52 chromosomes derived from unrelated individuals of European descent. We found that 12 polymorphic sites were present at $>10 \%$ allele frequency, including 11 SNPs and 1 tandem-repeat polymorphism. This variation resolved into three main haplotype clades spanning LTA (Fig. 1a).

We examined the same panel of 26 LCLs before and after stimulation with a mitogen (phorbol myristate acetate (PMA) plus iono-

a

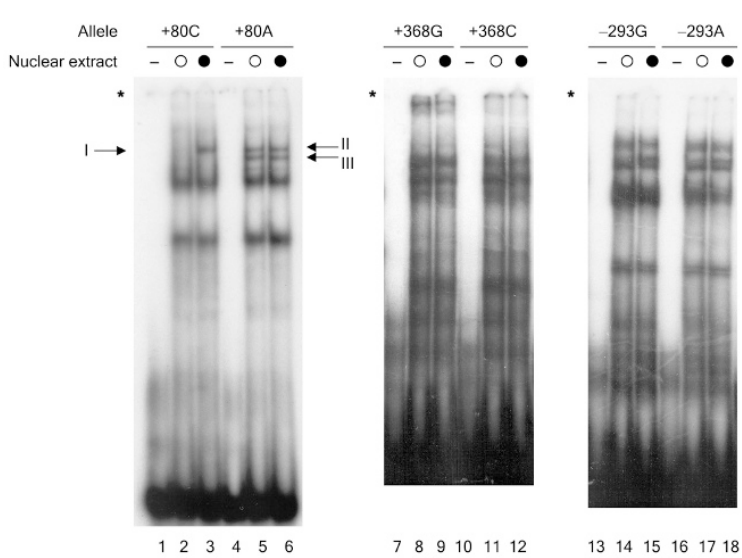

b

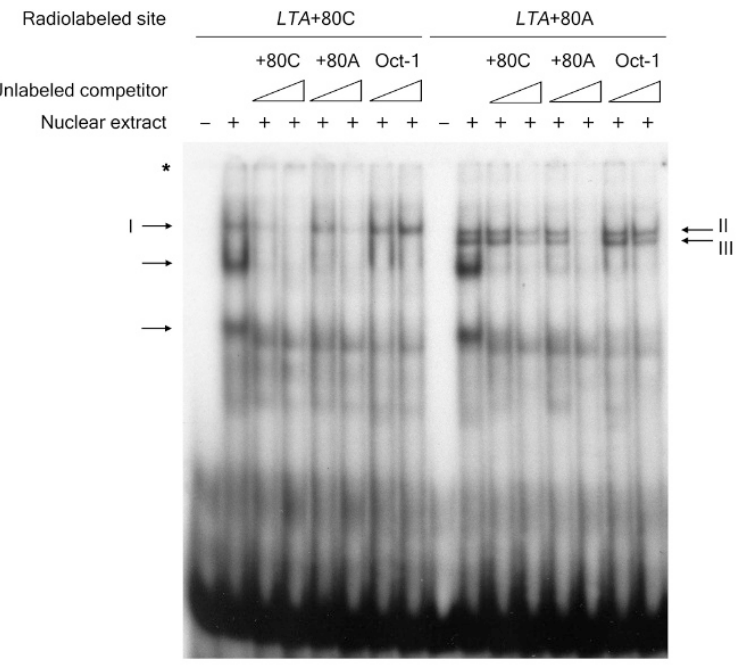

$\begin{array}{llllllllllllllll}1 & 2 & 3 & 4 & 5 & 6 & 7 & 8 & 9 & 10 & 11 & 12 & 13 & 14 & 15 & 16\end{array}$ mycin) that strongly induces LTA in this cellular model. In six replicate stimulation experiments, we determined LTA concentration in cell culture supernatants by ELISA 0, 24 and $48 \mathrm{~h}$ after stimulation and normalized this concentration to cell numbers at each time point. When we analyzed SNPs individually, LTA-294T, LTA-293A, $L T A+10 \mathrm{~A}, L T A+80 \mathrm{~A}, L T A+252 \mathrm{G}, L T A+368 \mathrm{C}, L T A+723 \mathrm{~A}, T N F-863 \mathrm{~A}$ and TNF-308A all showed significant associations with LTA production after stimulation and, to a lesser extent, in the resting state (Supplementary Table 1 online). This does not mean that all these SNPs are functional, as the high linkage disequilibrium at LTA distributes associations across the locus.

As a starting point in dissecting functionality, we carried out stepwise linear regression analysis using either all SNPs with $>10 \%$ allele frequency or the three main haplotype clades as predictive variables for induced LTA protein production (dependent variable). Analysis of individual SNPs identified $L T A+368 \mathrm{C}$ and $L T A+80 \mathrm{~A}$ (which in this data set always occur together) as the only significant predictive variables of LTA protein production (regression coefficient at $48 \mathrm{~h}$ after mitogen stimulation $(b)=143.9$ and standard error (s.e.) $=61, P=$ 0.027 ). These two SNPs uniquely define haplotype clade B, which was the only significant predictor when we entered the three haplotype clades as predictive variables of induced LTA production (at $24 \mathrm{~h}$ after mitogen stimulation, $b=129.6$ and s.e. $=61.8, P=0.048$; at $48 \mathrm{~h}$ after mitogen stimulation, $b=176.8$ and s.e. $=65.2, P=0.013$ ). Cells carrying haplotype clade B thus produce, on average, less LTA than other cells (Fig. 1b), consistent with our previous association of this clade with low polymerase II loading and transcript abundance ${ }^{17}$.

To explore possible molecular explanations, we investigated the haplotype-defining SNPs for effects on protein-DNA interactions in vitro using electrophoretic mobility shift assays (EMSAs). In addition to $L T A+368 \mathrm{C}$ and $L T A+80 A$, we studied $L T A-293 A$, which defines a large subset of clade $B$. When we incubated nuclear extracts from unstimulated and mitogen-stimulated LCLs with oligonucleotide probes spanning these SNPs, we observed allele-specific binding with probes spanning $L T A+80$ (Fig. 2a). A slowly migrating inducible complex bound to the $\mathrm{C}$ allele but not the A allele (complex I), whereas two constitutive complexes bound only to the A allele (complexes II and III). Competition EMSA experiments showed the specificity of allelic binding for these complexes (Fig. 2b). In contrast, LTA-293 showed no allele-specific differences in protein-DNA binding, and $L T A+368$ showed a very slowly migrating constitutive complex that seemed to be specific for the $\mathrm{C}$ allele and was not investigated further (Fig. 2a).

Several lines of evidence suggested that the allele-specific effects of the polymorphism at $L T A+80$ might involve the basic helix-loop-helix

Figure 2 Protein-DNA binding modulated by SNPs found on the functional haplotype. (a) EMSAs using nuclear extracts prepared from unstimulated (open circle) or mitogen-stimulated (filled circle) LCLs incubated with radioactively labeled probes spanning the sites of each of the SNPS in the two allelic forms. In this and all subsequent EMSAs, the location of the wells is indicated by an asterisk. (b) Competition EMSAs using radioactively labeled probes with either C (lanes 1-8) or A (lanes 9-16) at the LTA+80 SNP in a binding reaction with nuclear extract prepared from unstimulated LCLs. Complex I was specifically competed by $10 \times$ molar excess of unlabeled $L T A+80 C$ competitor probe, whereas unlabeled $\angle T A+80 A$ probe competed only at $100 \times$ molar excess. Complexes II and III were abolished by $100 \times$ molar excess of $\angle T A+80 A$, in contrast to unlabeled competitor $L T A+80 C$, which competed only weakly for binding. Molar excess of unlabeled probe matching an unrelated consensus binding site for Oct-1 did not compete for binding by complexes I, II or III, in contrast to a number of faster migrating complexes, which seem to be non-specific. 
Figure 3 In vitro recruitment of E-box binding proteins at $\angle T A+80 A$.

(a) Competition EMSAs using either a radioactively labeled probe matching $L T A+80 A$ or a consensus E-box binding element ( $\mu \mathrm{E} 5$ ) with nuclear extract prepared from unstimulated LCLs in the presence of $10 \times$ and $100 \times$ molar excess of unlabeled competitor probes as shown. (b) Supershift EMSAs using radioactively labeled probe spanning LTA+80A with nuclear extract prepared from unstimulated LCLs incubated in the presence of antibodies as indicated. Unstimulated nuclear extracts were used in these experiments as complexes II and III were present constitutively. a

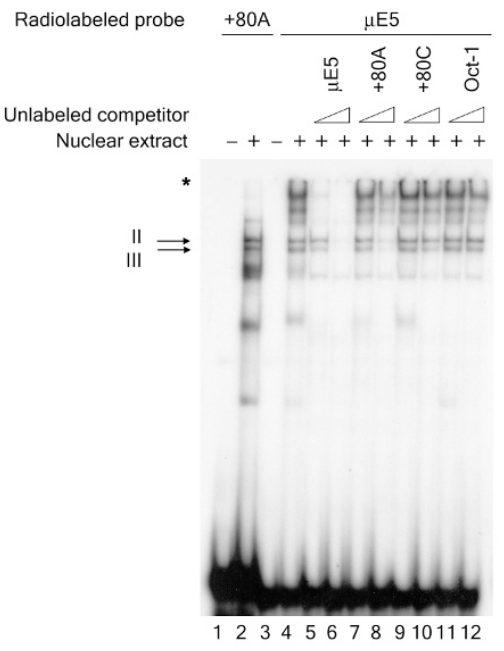

b

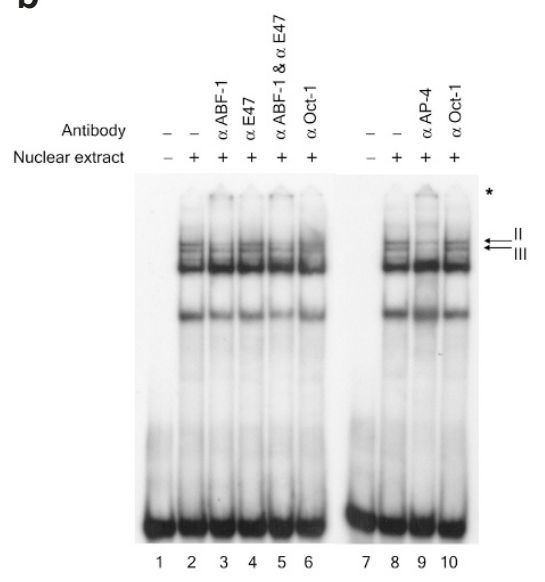

(bHLH) family of transcription factors. First, the sequence spanning the SNP at +80 shows homology with the E2-box consensus motif bound by class A bHLH proteins (CAGCTG) ${ }^{18}$ with a 1-bp mismatch (underlined) in the presence of an A at $L T A+80$ (CAGCAG), or a 2-bp mismatch with a $\mathrm{C}$ at $L T A+80$ (CEGCAG). When a probe matching a classical E-box binding element from the immunoglobulin heavychain (IgH) intronic enhancer ${ }^{19}, \mu \mathrm{E} 5$, was radioactively labeled and used in an EMSA with nuclear extracts from unstimulated LCLs, we observed two protein-DNA complexes that comigrated with complexes II and III observed with $L T A+80 \mathrm{~A}$ (Fig. 3a). The comigrating complexes binding to the radioactively labeled $\mu \mathrm{E} 5$ probe were competed away by molar excess of unlabeled probe matching $L T A+80 \mathrm{~A}$ but only weakly by $L T A+80 \mathrm{C}$ (Fig. 3a).

Antibody studies of the allele-specific complexes II and II identified on EMSA as binding to the sequence spanning $L T A+80 \mathrm{~A}$ provided preliminary evidence that at least two E-box proteins were present (Fig. 3b). Complex II was supershifted on EMSA using nuclear extracts from LCLs in the presence of antibodies to ABF-1. ABF-1 is a class II bHLH family member recently identified in LCLs that is specifically expressed in lymphoid tissue ${ }^{20}$. We found no evidence of supershift with antibodies to the usual heterodimeric partner of ABF-1, the bHLH protein E47. Complex III was supershifted with antibodies to AP-4, a member of the bHLH-Zip subgroup of Ebox binding proteins ${ }^{21}$. These data indicate that at least two E-box proteins show allele-specific recruitment to $L T A+80 \mathrm{~A}$ in vitro using crude nuclear extracts.

Figure 4 Differential binding of in vitro expressed E-box binding proteins. We analyzed total protein extracts from COS-7 cells transfected with either an empty vector (V) or with plasmids expressing ABF-1, E47 or AP-4 by EMSA. Control lanes show, in order, radioactively labeled probe alone and radioactively labeled probe incubated with protein extracts from nontransfected cells. (a) Protein extracts from COS-7 cells transfected with E47 and ABF-1 expression plasmids singly or together and incubated with a radioactively labeled probe for consensus E-box binding element, $\mu \mathrm{E} 5$. (b) Protein extracts from COS-7 cells transfected with E47 and ABF-1 expression plasmids singly or together and incubated with radioactively labeled probe spanning $L T A+80$ with either $\mathrm{C}$ or $\mathrm{A}$ at the site of the SNP. (c) Protein extracts from COS-7 cells transfected with AP-4 expression plasmid analyzed for binding to radioactively labeled probes for consensus E-box binding element, $\mu \mathrm{E} 5$, or probes spanning $L T A+80$ with either $\mathrm{C}$ or $\mathrm{A}$ at the site of the SNP.
To examine further allele-specific binding by candidate E-box binding proteins, we expressed ABF-1, E47 and AP-4 in COS-7 cells. We incubated protein extracts with radioactively labeled oligoduplexes corresponding to sites $L T A+80 \mathrm{C}, L T A+80 \mathrm{~A}$ and $\mu \mathrm{E} 5$, and analyzed them using EMSAs (Fig. 4a,b). For ABF-1 expressed alone, we observed no binding to any of the probes, consistent with previous observations that ABF-1 normally binds as a heterodimer ${ }^{20}$. In contrast, E47, which is capable of binding as a homodimer ${ }^{22}$, bound the consensus $\mu \mathrm{E} 5$ probe and, more weakly, the $L T A+80 \mathrm{~A}$ probe; no bind-

\section{a}

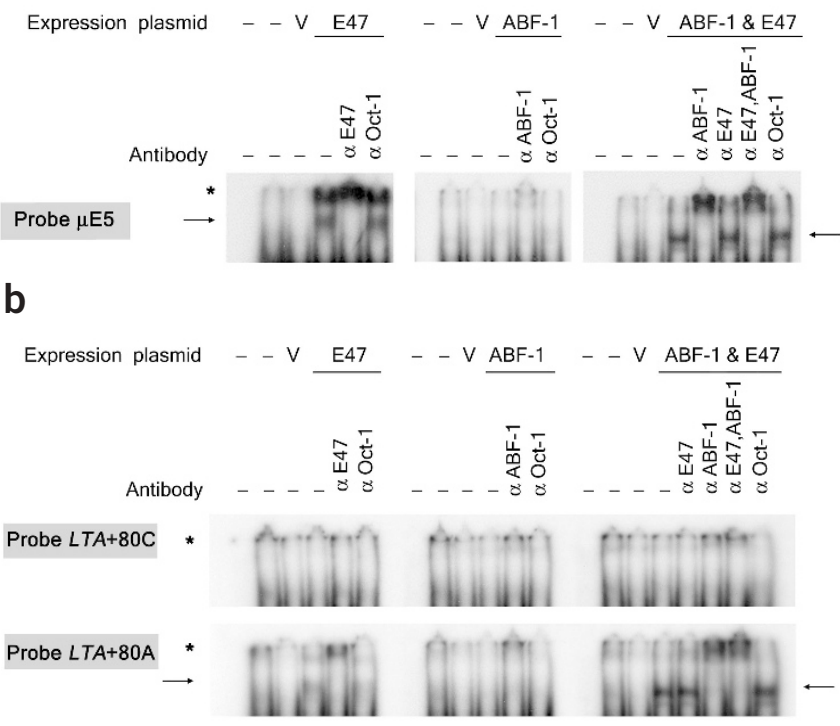

C

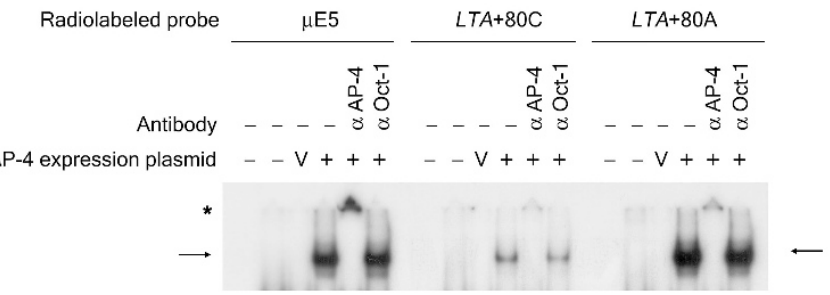


Figure 5 Allele-specific modulation of gene expression by ABF-1 and E47. We transiently transfected COS-7 cells with pGL3.prom reporter gene alone or with pGL3.prom reporters containing a 20-bp DNA fragment matching the LTA $5^{\prime}$ UTR (nucleotides +72 to +91 ) bearing $\mathrm{C}$ or $\mathrm{A}$ at the site of the $L T A+80$ SNP placed upstream of the SV40 promoter (designated pGL3. prom $L T A+80 C$ and pGL3.prom $\angle T A+80 A$, respectively). We transfected increasing amounts of empty expression vector or expression vectors for ABF-1 and E47 with the reporter constructs, including pBSK as required to keep the total amount of plasmid transfected constant $(0.5 \mu \mathrm{g})$. The mean \pm s.e.m. of five independent transient transfection experiments are shown together with paired $t$-tests comparing constructs $\mathrm{pGL}$. prom $\angle T A+80 \mathrm{~A}$ and pGL3. prom $L T A+80$ C (NS, $P>0.05 ;{ }^{*} P=0.02 ;{ }^{*} P=0.005 ;{ }^{* *} P=0.003$ ). Results are shown for pGL3. prom LTA constructs relative to pGL3. prom using values derived from luciferase activity normalized to Renilla and expressed relative to empty expression plasmid. When we transfected E47 expression construct in the absence of ABF-1, we found no difference between levels of activity of the two LTA constructs using $0.0625,0.125$ or $0.25 \mu \mathrm{g}$ of E47 expression plasmid. There was, however, a small but significant difference between LTA constructs using $0.5 \mu \mathrm{g}$ of $E 47$ expression plasmid ( $P=0.043$ in a paired $t$-test of five independent transfection experiments): mean luciferase activity of pGL3.prom $L T A+80 A$ relative to pGL3.prom was 1.27 (s.d. 0.16) compared with 1.00 (s.d. 0.28) for pGL3.promLTA+80C relative to pGL3.prom. For pGL3. prom transfected alone into $4 \times 10^{5}$ COS-7 cells, mean luminometer values of 2,398 light units were obtained.

ing was seen for $L T A+80 \mathrm{C}$. When we coexpressed ABF-1 with E47, we observed binding to $\mu \mathrm{E} 5$ and $L T A+80 \mathrm{~A}$ but no binding to the $L T A+80 \mathrm{C}$ allele probe. The complex was supershifted in the presence of antibody to ABF-1 but not E47. The specificity of the antibody to ABF-1 was resolved by western blotting (see Supplementary Fig. 1 online). AP-4 bound strongly to both the $\mu \mathrm{E} 5$ and $L T A+80 \mathrm{~A}$ allele probes but only very weakly to the $L T A+80 \mathrm{C}$ allele probes (Fig. $4 \mathrm{c}$ ). These data indicate that the sequences spanning $L T A+80$ show allelespecific differences in their ability to bind E-box proteins in vitro.

We then investigated whether the allele-specific recruitment of Ebox binding proteins observed in vitro modulated levels of gene expression. We carried out transient transfection assays in which these proteins were overexpressed in the presence of constructs bearing the $L T A+80$ SNP. We engineered reporter gene constructs in which 20-bp DNA fragments of the LTA $5^{\prime}$ untranslated region (UTR) corresponding to each of the two allelic variants of the LTA +80 SNP were placed upstream of an SV40 promoter in a pGL3 luciferase reporter. We then transiently transfected these constructs into COS-7 cells. When

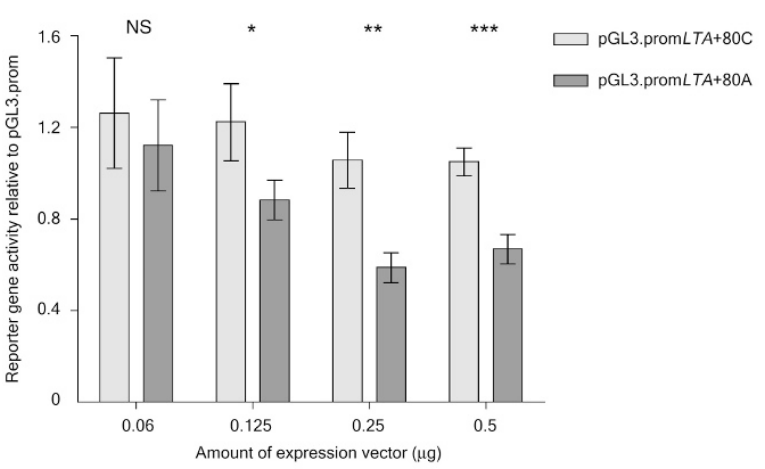

cotransfected with ABF-1 and E47, the constructs bearing $L T A+80 \mathrm{~A}$ relative to the SV40 promoter alone had significantly lower reporter gene activity than did the $L T A+80 \mathrm{C}$ constructs (Fig. 5). We observed a dose-dependent effect, with increasing repression when we cotransfected increasing amounts of ABF-1/E47 expression plasmids (Fig. 5). We found no significant difference between LTA reporter gene plasmids $(P>0.05)$ at any amount of AP-4 expression plasmid cotransfected $(0.0625-0.5 \mu \mathrm{g})$. These data provided evidence that ABF-1 has the capacity to modulate reporter gene expression in an allele-specific manner, in contrast to AP-4, which showed higher DNA binding affinity in the presence of the $L T A+80 \mathrm{~A}$ allele but no difference in expression after cotransfection.

The functional data from assays of protein-DNA binding demonstrated the capacity in vitro for E-box binding proteins ABF-1 and AP-4 to bind in an allele-specific manner, modulated by the $L T A+80$ polymorphism. To seek evidence that these E-box binding proteins were recruited in vivo, we investigated binding of ABF-1 and AP-4 at the LTA promoter or 5' UTR by chromatin immunoprecipitation (ChIP) using a a

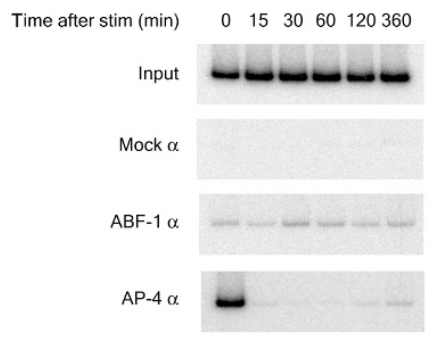

b

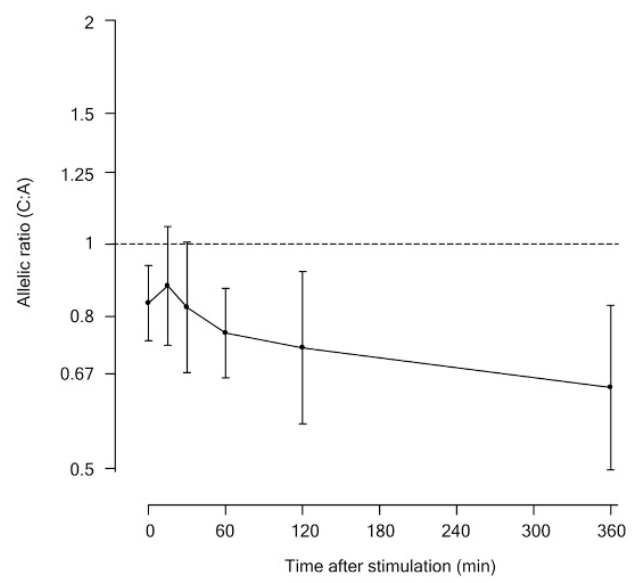

Figure 6 Allele-specific occupancy by ABF-1 in vivo. (a) We analyzed recruitment of ABF-1 and AP-4 at the LTA promoter and 5' UTR (nucleotides +171 to -46 ) by ChIP using a LCL stimulated with ionomycin and PMA (lanes 1-6) for the times shown. Input shows the products of PCR reactions containing $0.5 \%$ of total amount of chromatin used in immunoprecipitation reactions. Mock immunoprecipitation reactions using irrelevant control antibodies (against $T$ antigen pAb101) together with immunoprecipitations done using antibodies specific for ABF-1 or AP-4 are shown. (b) Allele-specific loading of ABF-1 analyzed for $L T A+80$ SNP by PE-MS. We stimulated four cell lines heterozygous with respect to $L T A+80$ SNP with ionomycin and PMA. The overall geometric mean ratios ( $\pm 95 \%$ c.i.) of $A B F-1$ loading between the $C$ and $A$ alleles are shown. 
LCL heterozygous with respect to $L T A+80$ (Fig. 6a). ABF-1 and AP-4 were recruited to the region, with AP-4 present constitutively but not after mitogen stimulation. We then carried out haplotype-specific ChIP (haploChIP) using the $L T A+80$ polymorphism as a marker to analyze allele-specific occupancy by ABF- 1 and AP-4 in each of four B cell lines heterozygous with respect to $L T A+80$. We previously showed that accurate quantification of relative allelic abundance can be assayed by this method using primer extension-mass spectrometry (PE-MS) ${ }^{17}$. We generated standard curves for the $L T A+80$ polymorphism by analyzing differing known input ratios of plasmid DNA for the two allelic forms from 1:4 to 4:1. This analysis showed a strong linear relationship $\left(\mathrm{r}^{2}=\right.$ $0.96)$ with the ratios observed by PE-MS. We then analyzed allele-specific occupancy by ABF-1 using haploChIP. This analysis showed progressively more binding by ABF- 1 to the $L T A+80 \mathrm{~A}$ allele over time after mitogen stimulation (one-way analysis of variance test for linear trend over time $P=0.004, \mathrm{r}^{2}=0.349$; Fig. $6 \mathbf{b}$ ). In contrast, the constitutive binding that we observed for AP-4 showed no significant allelic difference, with a mean ratio $L T A+80 \mathrm{C}: L T A+80 \mathrm{~A}$ of 1.08 (95\% confidence interval (c.i.) $=0.56-1.25)$.

Phenotypic variation in LTA production has previously been correlated with the $L T A+252$ polymorphism ${ }^{4,13,14,16}$. Here we identified nine different polymorphisms that correlate with LTA protein production. When LTA sequence variation is analyzed in detail using multivariate statistics, this complex pattern of association can be reduced to the effects of a specific low-producing haplotype clade. This commonly occurring haplotype is uniquely defined by two markers, one of which, $L T A+80$, was found in vitro to modulate allele-specific binding of two E-box binding proteins, ABF-1 and AP-4, in the presence of the $L T A+80 \mathrm{~A}$ allele. ABF- 1 is a recently described transcription factor that has been particularly associated with lymphoid tissue ${ }^{20,23}$, raising the possibility that the functional effects of the $L T A+80$ polymorphism are tissue-specific. ABF-1 repressed transcription in our reporter gene model when the $L T A+80 \mathrm{~A}$ allele was present, consistent with previous reports that it acts as a transcriptional repressor ${ }^{20,23}$, whereas AP- 4 had no effect on allele-specific expression.

The haploChIP method provides a powerful new tool for investigating whether regulatory polymorphisms alter specific DNA-protein interactions in vivo (i.e., to investigate polymorphisms in their natural chromatin structure in a living cell). The method was initially developed to assess the effects of allelic variation on polymerase II binding, as a surrogate marker of gene expression ${ }^{17}$. Here we show how it may be used to acquire specific information about the mode of action of a putative regulatory polymorphism. When we tested the hypothesis generated from our in vitro data that the $L T A+80$ polymorphism modulates binding of ABF- 1 and AP-4, we found allele-specific differences in binding in vivo by haploChIP for ABF-1 whereas AP-4 showed constitutive binding that was unaffected by the polymorphism. The AP-4 signal on chromatin immunoprecipitation may emanate from a nonpolymorphic binding site in the same vicinity, such as the E-box motif that is located 317-312 nucleotides upstream of the transcriptional start site, which would be present on the same amplified DNA fragments after sonication as these are typically $1 \mathrm{~kb}$ in length.

Can the functional effects of the $L T A+80 \mathrm{~A}$ allele explain the reported epidemiological associations between polymorphisms in the LTA region and susceptibility to common diseases ${ }^{4-12}$ ? Our analysis of haplotype structure in 52 chromosomes of European origin confirmed high linkage disequilibrium across the $10-\mathrm{kb}$ region. The $L T A+80 \mathrm{~A}$ allele was present on $70 \%$ of chromosomes analyzed. The $L T A+252 \mathrm{G}$ allele was not present on any of the chromosomes that carried the $L T A+80 A$ allele but was present on $57 \%$ of chromosomes that did not. Thus, the significant association that we observed between the
LTA $+252 \mathrm{G}$ allele and high LTA protein production could entirely be explained, when examined by multivariate analysis, by the ability of the $L T A+80 \mathrm{~A}$ allele to suppress LTA production. Analysis of larger data sets or different experimental models may identify other functional variants at the LTA locus ${ }^{4}$, but based on these data, we can at least state that $L T A+80 \mathrm{~A}$ is a good functional candidate to explain the reported disease associations of the $L T A+252 \mathrm{G}$ allele, and this needs to be addressed in future epidemiological studies.

\section{METHODS}

Plasmids and haplotype construction. To generate the pGL3.promLTA+80C and pGL3.promLTA+80A constructs, we designed oligonucleotides spanning the $L T A+80$ site (sequences are available on request), which we annealed and cloned into a KpnI-BglII-digested dephosphorylated pGL3-promoter vector (Promega) upstream of the SV40 promoter. We verified all constructs by DNA sequencing. Expression plasmids used were pHBA.FLAG-ABF-1 (ref. 20), pHBA-E47 (ref. 20), pHBAPr-1-neo ${ }^{24}$ and pcDNA3-AP-4MD ${ }^{25}$. To generate the haplotypic data for the panel of LCLs, we carried out long-range PCR from genomic DNA, cloning and sequencing as previously described ${ }^{17}$ and used polyphred alignment to create haplotypes from each hemizygous clone. Contig locations (GenBank accession number Y14768) for the SNPs described were as follows: LTA-294, 47622; LTA-293, 47621; LTA+10, 47318; LTA+80, 47248; $L T A+252,47076 ; L T A+368,46960 ; L T A+438,46890 ; L T A+495,46833$; LTA+723, 46605; TNF-1031, 45081; TNF-863, 44913; TNF-308, 44358.

Nuclear extracts, EMSAs, western blotting and antibodies. We annealed oligonucleotide probes (sequences are available on request) and used Klenow to radioactively label the resulting oligoduplexes $L T A+80 \mathrm{C}, L T A+80 \mathrm{~A}$, LTA+368G, LTA+368C, LTA-293G, LTA-293A, Oct-1 and $\mu$ E5 with $\left[\alpha^{32} \mathrm{P}\right] \mathrm{dCTP}$ (Amersham Pharmacia Biotech). We prepared nuclear extracts from $20 \times 10^{6}$ LCLs as previously described ${ }^{26,27}$. We mixed $2 \mu \mathrm{g}$ of nuclear extract with $0.2-0.5 \mathrm{ng}$ of labeled probe $\left(1-5 \times 10^{4}\right.$ c.p.m. $)$ in a $10-\mu$ reaction containing 12 mM HEPES buffer (pH 7.8), 100 mM NaCl, 1 mM EDTA, $1 \mathrm{mM}$ EGTA, 12\% glycerol and $0.5 \mu \mathrm{g}$ poly (dA-dT) (Amersham Pharmacia Biotech) and incubated it at room temperature for $20 \mathrm{~min}$. We analyzed the reaction by electrophoresis in a nondenaturing polyacrylamide gel at $4{ }^{\circ} \mathrm{C}$ in $0.5 \times$ TBE buffer. For supershift analysis, we preincubated nuclear extract with appropriate antiserum at room temperature for $20 \mathrm{~min}$ before adding the radioactively labeled probe. We used the following antibodies for supershift studies: ABF-1 (sc9555) and Oct-1 (sc232; Santa Cruz); human monoclonal antibody to E47/E12 (554199; BD Biosciences); AP4 (ref. 28). We transfected COS-7 cells with constructs expressing pHBA.FLAG-ABF-1, pHBA-E47, pHBAPr-1-neo, pcDNA3-AP-4MD and pcDNA3 and then prepared total protein extracts by lysing cells in lysis buffer (20 mM Tris- $\mathrm{HCl}(\mathrm{pH}$ 8), $300 \mathrm{mM} \mathrm{NaCl}, 0.1 \%$ Nonidet P-40, 10\% glycerol) supplemented with Complete protease inhibitors (Boeringher Mannheim). We carried out western blotting for total protein in COS-7 cell extracts by fractionation on a $4-12 \%$ NuPage Bis-Tris polyacrylamide gel (Invitrogen) in $1 \times$ MOPS buffer, followed by transfer to a PVDF membrane (Invitrogen) and incubation with primary antibody in $4 \%$ dried milk powder with $0.1 \%$ Tween in phosphate-buffered saline. We used primary antibodies to ABF-1 (sc9555) at 1:500 dilution and to $\beta$-tubulin 1 (T-7816; Sigma) at $1: 1,000,000$ dilution. We visualized bands by chemiluminescence with horseradish peroxidase-conjugated rabbit antibody to goat and goat antibody to mouse, respectively, using ECL Plus (Amersham).

Cell culture, transfections and luciferase assay. We obtained EBV-transformed B cell lines and cultured them as described previously ${ }^{17}$. We stimulated cells with $200 \mathrm{nM}$ PMA (Sigma) and $125 \mathrm{nM}$ ionomycin (Sigma) unless otherwise stated. We assayed LTA concentration in cell culture supernatants in duplicate using an LTA ELISA kit (AMS Biotechnology) according to the manufacturer's instructions. We analyzed data by linear regression using SPSS software. We cultured COS-7 cells in Dulbecco's modified Eagle medium supplemented with 10\% fetal bovine serum, $100 \mathrm{U} \mathrm{ml}^{-1}$ penicillin, $100 \mathrm{mg} \mathrm{ml}^{-1}$ streptomycin, $0.2 \mathrm{mM} \mathrm{L-gluta-}$ mine at $37{ }^{\circ} \mathrm{C}$ in $5 \%$ humidified $\mathrm{CO}_{2}$. We used Polyfect (Qiagen) for transient transfection of expression constructs, with and without reporter gene constructs, into COS-7 cells following the manufacturer's protocol. We transfected $4 \times 10^{5}$ 
COS-7 cells with $1.5 \mu \mathrm{g}$ of total DNA $(0.5 \mu \mathrm{g}$ of expression plasmid or pBSK, 0.167 $\mu \mathrm{g}$ of pGL3 reporter and $0.833 \mu \mathrm{g}$ of pRL.TK) and collected them after $48 \mathrm{~h}$. We cotransfected pRL.TK to normalize for transfection efficiency. We kept the total amount of DNA used in each transfection experiment constant by addition of pBSK. We lysed cells in $500 \mu \mathrm{l}$ of $1 \times$ lysis buffer (Promega) and analyzed $10 \mu \mathrm{l}$ using the Dual-Luciferase reporter assay kit (Promega) and a Turner luminometer model 20 (Promega) following the manufacturer's protocol.

Chromatin immunoprecipitation. We prepared chromatin from $5 \times 10^{8}$ cells, sonicated it and purified it on a cesium-chloride gradient as previously described ${ }^{17}$. We incubated Dynabeads M-280 precoated with appropriate sheep antibodies to mouse or to rabbit IgG overnight with primary antibodies at $4{ }^{\circ} \mathrm{C}$ in phosphate-buffered saline containing $5 \mathrm{mg} \mathrm{ml}^{-1}$ bovine serum albumin (Sigma). To generate magnetic beads coated with antibody to goat, we incubated Dynabeads M-280 with biotinylated antibodies to goat IgG (227-AB2G2; AMS Biotechnology) for $30 \mathrm{~min}$ at room temperature and then incubated washed beads with primary antibody as described. We used specific antibodies against ABF-1 and AP-4 as described for supershift analysis and also did mock antibody controls using antibody to actin (A2066; Sigma). After washing to remove unbound antibody, we incubated antibody beads overnight at $4{ }^{\circ} \mathrm{C}$ with $50 \mu \mathrm{g}$ of chromatin in $1 \times$ RIPA buffer ${ }^{29}$ containing $1 \times$ complete protease inhibitors (Roche) and $5 \mu \mathrm{g} \mathrm{ml}^{-1}$ pepstatin (Roche) on a nutator. We washed bead-chromatin immunoprecipitations in $1 \times$ RIPA buffer containing $500 \mathrm{mM}$ $\mathrm{LiCl}$ (Sigma) eight times with a final wash in Tris-EDTA buffer. We resuspended beads in $120 \mu \mathrm{l}$ of Tris-EDTA digestion buffer containing 1\% SDS, incubated them at $65^{\circ} \mathrm{C}$ for $10 \mathrm{~min}$, pelleted beads by centrifugation and incubated the supernatant at $65^{\circ} \mathrm{C}$ overnight to reverse cross-links. We added proteinase $\mathrm{K}$ to $200 \mu \mathrm{g} \mathrm{ml}^{-1}$ final concentration, incubated at $37^{\circ} \mathrm{C}$ for $2 \mathrm{~h}$, extracted the DNA using phenol and chloroform and precipitated it with ethanol in the presence of $20 \mu \mathrm{g}$ of glycogen. We resuspended pellets in $30 \mu \mathrm{l}$ of Tris-EDTA containing $10 \mu \mathrm{g}$ of RNase A, incubated them at $37^{\circ} \mathrm{C}$ for $1 \mathrm{~h}$, purified the reactions by spin column and assayed them by semiquantitative $\mathrm{PCR}^{30}$. We amplified the LTA region between -46 and +171 relative to the transcriptional start site (primer sequences available on request) with PCR cycling parameters of $96^{\circ} \mathrm{C}$ for 1 min then 5 cycles of $96^{\circ} \mathrm{C}$ for $35 \mathrm{~s}, 68^{\circ} \mathrm{C}$ for $45 \mathrm{~s}, 72^{\circ} \mathrm{C}$ for $35 \mathrm{~s}$; then 20 cycles of $96^{\circ} \mathrm{C}$ for $35 \mathrm{~s}, 64^{\circ} \mathrm{C}$ for $45 \mathrm{~s}, 72^{\circ} \mathrm{C}$ for $40 \mathrm{~s}$; and then a final 6 cycles of $96{ }^{\circ} \mathrm{C}$ for $35 \mathrm{~s}, 56^{\circ} \mathrm{C}$ for $60 \mathrm{~s}, 72^{\circ} \mathrm{C}$ for $90 \mathrm{~s}$. We carried out haploChIP analysis using PE-MS as previously described ${ }^{17}$. We carried out first-round amplification with primers and cycling parameters as described for semiquantitative PCR except we used 27 cycles rather than 20 cycles annealing at $64{ }^{\circ} \mathrm{C}$. We carried out primer extension for $L T A+80$ (extension primer sequence available on request) using MassEXTEND (Sequenom) as described previously ${ }^{17}$. We immunoprecipitated each cell line twice and analyzed the products of immunoprecipitation by four independent PCR reactions, which were in turn each spotted in quadruplicate on the chip before PE-MS. As we have observed previously for certain $\mathrm{SNPs}^{17}$, we found a systematic allelic difference in PE-MS signal intensity for $L T A+80$ when we compared observed ratios with a range of known input ratios (from 1:4 to 4:1). ChIP sample ratios given were normalized by the input ratios.

\section{Note: Supplementary information is available on the Nature Genetics website.}

\section{ACKNOWLEDGMENTS}

We thank C. Murre for the expression plasmids pHBA.FLAG-ABF-1, pHBA-E47 and pHBAPr-1-neo; A. Aranburu for the expression plasmid pcDNA3-AP-4MD; and R. Gaynor for antibody to AP4. This work was supported financially by the Medical Research Council, UK.

\section{COMPETING INTERESTS STATEMENT}

The authors declare that they have no competing financial interests.

Received 4 November 2003; accepted 18 February 2004

Published online at http://www.nature.com/naturegenetics/

1. Paul, N.L. \& Ruddle, N.H. Lymphotoxin. Annu. Rev. Immunol. 6, 407-438 (1988),

2. Roach, D.R. et al. Secreted lymphotoxin-alpha is essential for the control of an intra- cellular bacterial infection. J. Exp. Med. 193, 239-246 (2001).

3. Engwerda, C.R. et al. Locally up-regulated lymphotoxin alpha, not systemic tumor necrosis factor alpha, is the principle mediator of murine cerebral malaria. J. Exp. Med. 195, 1371-1377 (2002).

4. Ozaki, K. et al. Functional SNPs in the lymphotoxin-alpha gene that are associated with susceptibility to myocardial infarction. Nat. Genet. 32, 650-654 (2002).

5. Albuquerque, R.V. et al. Association of polymorphisms within the tumour necrosis factor (TNF) genes and childhood asthma. Clin. Exp. Allergy 28, 578-584 (1998).

6. Moffatt, M.F. \& Cookson, W.O. Tumour necrosis factor haplotypes and asthma. Hum. Mol. Genet. 6, 551-554 (1997).

7. Stuber, F., Petersen, M., Bokelmann, F. \& Schade, U. A genomic polymorphism within the tumor necrosis factor locus influences plasma tumor necrosis factor-alpha concentrations and outcome of patients with severe sepsis. Crit. Care Med. 24 381-384 (1996).

8. Majetschak, M. et al. Relation of a TNF gene polymorphism to severe sepsis in trauma patients. Ann. Surg. 230, 207-214 (1999).

9. Demeter, J. et al. Polymorphism of the tumor necrosis factor-alpha and lymphotoxinalpha genes in chronic lymphocytic leukaemia. Br. J. Haematol. 97, 107-112 (1997).

10. Shimura, T. et al. The study of tumor necrosis factor beta gene polymorphism in lung cancer patients. Cancer 73, 1184-1188 (1994).

11. Yamaguchi, E., Itoh, A., Hizawa, N. \& Kawakami, Y. The gene polymorphism of tumor necrosis factor-beta, but not that of tumor necrosis factor-alpha, is associated with the prognosis of sarcoidosis. Chest 119, 753-761 (2001).

12. Badenhoop, K. et al. Tumor necrosis factor beta polymorphisms in Graves' disease. J. Clin. Endocrinol. Metab. 74, 287-291 (1992).

13. Messer, G. et al. Polymorphic structure of the tumor necrosis factor (TNF) locus: an Ncol polymorphism in the first intron of the human TNF-beta gene correlates with a variant amino acid in position 26 and a reduced level of TNF- beta production. J. Exp. Med. 173, 209-219 (1991)

14. Lee, S.H. et al. Decreased tumour necrosis factor-beta production in TNFB*2 homozygote: an important predisposing factor of lupus nephritis in Koreans. Lupus $\mathbf{6}$, 603-609 (1997).

15. Pociot, F. et al. Association of tumor necrosis factor (TNF) and class II major histocompatibility complex alleles with the secretion of TNF-alpha and TNF-beta by human mononuclear cells: a possible link to insulin- dependent diabetes mellitus. Eur. J. Immunol. 23, 224-231 (1993).

16. Whichelow, C.E., Hitman, G.A., Raafat, I., Bottazzo, G.F. \& Sachs, J.A. The effect of TNF*B gene polymorphism on TNF-alpha and -beta secretion levels in patients with insulin-dependent diabetes mellitus and healthy controls. Eur. J. Immunogenet. $\mathbf{2 3}$ 425-435 (1996).

17. Knight, J.C., Keating, B.J., Rockett, K.A. \& Kwiatkowski, D.P. In vivo characterization of regulatory polymorphisms by allele-specific quantification of RNA polymerase loading. Nat. Genet. 33, 469-475 (2003).

18. Dang, C.V., Dolde, C., Gillison, M.L. \& Kato, G.J. Discrimination between related DNA sites by a single amino acid residue of Myc-related basic-helix-loop-helix proteins. Proc. Natl. Acad. Sci. USA 89, 599-602 (1992).

19. Ephrussi, A., Church, G.M., Tonegawa, S. \& Gilbert, W. B lineage-specific interactions of an immunoglobulin enhancer with cellular factors in vivo. Science 227, 134-140 (1985)

20. Massari, M.E. et al. Characterization of ABF-1, a novel basic helix-loop-helix transcription factor expressed in activated B lymphocytes. Mol. Cell. Biol. 18, 3130-3139 (1998)

21. Mermod, N., Williams, T.J. \& Tjian, R. Enhancer binding factors AP-4 and AP-1 act in concert to activate SV4O late transcription in vitro. Nature 332, 557-561 (1988).

22. Shen, C.-P. \& Kadesch, T. B-cell-specific DNA binding by an E47 homodimer. Mol. Cell. Biol. 15, 4518-4524 (1995).

23. Wong, J. et al. Characterization of a basic helix-loop-helix protein, ABF-1: nuclear localization, transcriptional properties, and interaction with Id-2. DNA Cell. Biol. 20, 465-471 (2001)

24. Gunning, P., Leavitt, J., Muscat, G., Ng, S.Y. \& Kedes, L. A human beta-actin expression vector system directs high-level accumulation of antisense transcripts. Proc. Natl. Acad. Sci. USA 84, 4831-4835 (1987).

25. Aranburu, A., Carlsson, R., Persson, C. \& Leanderson, T. Transcription factor AP-4 is a ligand for immunoglobulin-kappa promoter E-box elements. Biochem. J. 354 431-438 (2001).

26. Schreiber, E., Matthias, P., Muller, M.M. \& Schaffner, W. Rapid detection of octamer binding proteins with 'mini-extracts', prepared from a small number of cells. Nucleic Acids Res. 17, 6419 (1989)

27. Udalova, I.A., Knight, J.C., Vidal, V., Nedospasov, S.A. \& Kwiatkowski, D. Complex NF-kappaB interactions at the distal tumor necrosis factor promoter region in human monocytes. J. Biol. Chem. 273, 21178-21186 (1998).

28. Ou, S.H., Garcia-Martinez, L.F., Paulssen, E.J. \& Gaynor, R.B. Role of flanking E box motifs in human immunodeficiency virus type 1 TATA element function. J. Virol. 68 7188-7199 (1994).

29. Nissen, R.M. \& Yamamoto, K.R. The glucocorticoid receptor inhibits NFkappaB by interfering with serine-2 phosphorylation of the RNA polymerase II carboxy-terminal domain. Genes Dev. 14, 2314-2329 (2000).

30. Takahashi, Y., Rayman, J.B. \& Dynlacht, B.D. Analysis of promoter binding by the $\mathrm{E} 2 \mathrm{~F}$ and $\mathrm{pRB}$ families in vivo: distinct E2F proteins mediate activation and repression. Genes Dev. 14, 804-816 (2000). 